\title{
Effects of seasonal change in rugby league on the incidence of injury
}

\author{
L Hodgson Phillips, P J Standen, M E Batt
}

Department of Orthopaedic and Accident Surgery, Queen's Medical Centre, University Hospital, Nottingham NG7 2UH, United Kingdom

L Hodgson Phillips

Department of Learning Disabilities, University Hospital, Queen's Medical Centre, Nottingham P J Standen

Centre for Sports Medicine, Department of Orthopaedic and Accident Surgery, University Hospital, Queen's Medical Centre

M E Batt

Correspondence to: Lisa Hodgson Phillips.

Accepted for publication 13 November 1997

\begin{abstract}
Objectives-To assess the effects that the recent seasonal change in rugby league from winter to summer has had on the incidence of injury.

Methods-All injuries that occurred during games and training were recorded throughout four consecutive seasons (three winter, one summer) for the first team squad of a British professional rugby league club. Data included nature of injury, days lost as the result of injury, player position, month, and season.

Results-An increasing incidence of injury over the four seasons was observed, with the summer season having an incidence almost double that of the first winter season recorded (696.8 per 1000 hours and 363.55 per 1000 hours respectively). The severity of injury was shown to decline over the four seasons. Most injuries were incurred in matches $(74.9 \%)$ rather than during training $(25.1 \%)$.

Conclusions-The findings show an increasing incidence of injury in summer rugby but with decreased severity. It is not known if this is the result of playing back to back rugby seasons or is a consequence of a change in playing season and ground conditions.
\end{abstract}

(Br J Sports Med 1998;32:144-148)

Keywords: rugby league; injury incidence; winter; summer

Rugby league is a collision sport of skill, requiring a combination of muscular strength, stamina, and endurance. ${ }^{12}$ Tackling is one of the most important individual skills in the game, but endurance is also required as players may cover between 5000 and $7000 \mathrm{~m}$ during an 80 minute match. ${ }^{3}$ It may be expected that rugby league would have high injury rates, but research into this is very limited. It is not easy to compare incidence of injury in sport unless data are collected and analysed in comparable ways. Increasingly incidence rates in all sports are being reported as the number of injuries per 1000 hours played. An early Australian rugby league study on all injuries to three professional teams, over a one year period gave an incidence rate of 277.78 per 1000 hours. ${ }^{4}$ More recently, a prospective three year study of a professional South Sydney rugby league club gave an incidence of 44.9 per 1000 hours. The latter study only registered injuries that resulted in a subsequent game being missed, ${ }^{1}$ highlighting the issue of injury definition. A recent four year prospective study carried out in England on game injuries at one first division club gave an incidence rate of 114 per 1000 hours. ${ }^{5}$ Differences in data can be related to methodology of the studies.

In England, rugby league was a winter game with the season running from August to April. A condensed season from August 1995 to January 1996 marked the transition from winter to summer rugby, the first summer season running from March to August 1996. This change might be expected to influence the pattern of injuries because of the change from soft wet ground to hard dry ground. ${ }^{6}$ Intraseasonal variation has previously been explored, ${ }^{7-10}$ but no consensus has been reached. Some studies note proportionally more injuries towards the end of the season ${ }^{9}{ }^{10}$; others report the greatest proportion in early season. ${ }^{78}$

The summer season retained a schedule of one game a week; however, the condensed season saw games played more frequently. During the first three seasons studied, a maximum of two substitute players was allowed (four replacements); however, during the 1996 summer season, four substitute players were allowed (six replacements).

The purpose of this study is to assess the effects that the recent seasonal changes in rugby league have had on the incidence of injury. When examining these effects it is important to include injuries incurred during training. Training sessions, typically involving minimal body contact, were conducted four to five times a week during each season. This should minimise player injuries, and training injuries have been found to be uncommon ${ }^{1}$; however, the inclusion of these figures gives a more detailed picture of injuries in a rugby league season.

The start of the "new summer rugby" era provided an ideal opportunity to compile data. The study compares two "old" winter seasons with the only ever condensed season and the very first summer season.

\section{Methods}

During the 1993/1994, 1994/1995, 1995/1996 (all winter) and 1996 (summer) seasons, all injuries incurred during competitive games and training sessions in the first team squad of a British professional rugby league club were recorded by the team physiotherapist (LHP), who was in attendance at all games and training sessions throughout the four seasons. The method of recording injury data remained consistent throughout, the first three seasons' data being retrospective and the 1996 summer season prospective.

Injuries were recorded irrespective of whether the player missed a subsequent 
Table 1 Games played for one professional rugby league team over four consecutive seasons

\begin{tabular}{lccccr}
\hline \multirow{2}{*}{ Type of game } & Number played & & & \\
\cline { 2 - 6 } & $1993 / 1994$ & $1994 / 1995$ & $1995 / 1996$ & 1996 & Total \\
\hline Friendly & 1 & 1 & 1 & - & 3 \\
League & 30 & 30 & 20 & 20 & 100 \\
Regal Trophy & 1 & 1 & 1 & -1 & 4 \\
Challenge Cup & 3 & 4 & 23 & 21 & 115 \\
Total & 35 & 36 & & & \\
\hline
\end{tabular}

Table 2 Observed and expected injury totals for each season

\begin{tabular}{lllll}
\hline & $1993 / 1994$ & $1994 / 1995$ & $1995 / 1996$ & 1996 \\
\hline Observed & 220 & 231 & 216 & 253 \\
Expected & 280 & 288 & 184 & 168 \\
\hline
\end{tabular}

session/game. All injuries were identified on game day by the physiotherapist present or were reported by the player at the next training session. Any players who did not report, but whom the physiotherapist had seen for attention/advice after the game, were also recorded and followed up. An injury was defined as pain, discomfort, disability, or illness (new or recurrent) that the player acknowledged after participating in a rugby related activity/game. This also included any advice/ intervention sought from the medical team. The diagnosis and classification of injury was carried out by the physiotherapist in consultation with the club doctors and a team orthopaedic surgeon when appropriate.

To allow comparisons with other studies, ${ }^{11}$ the injuries were classified into transient (no games or training missed), minor (up to one game missed), moderate (two to four games missed), and severe (five or more games missed).

DATA RECORDED

For each injury the following information was recorded.

(1) The player, his position, age, and previous medical history. Each squad consisted of 22 players, except for the 1995/1996 season when each squad had 25 players, available for first team selection. Players not utilised and not injured may have played in the alliance division (second team) at some time. This provided a total sample of 44 players for whom injury data events were documented; some players were present throughout the four seasons.

(2) When the injury occurred (playing or training)

(3) The site and nature (type) of injury

(4) The month and season in which the injury occurred

(5) The number of days of training/playing missed as a result of the injury.
This paper reports seasonal effects on frequency and severity. Other data will be reported in subsequent publications.

ANALYSIS

The duration of each game was 80 minutes (1.33 hours). As there was one player for each of the 13 positions on the field at any one time, the injury exposure for the first team over the total 115 games for four seasons was calculated as 1988.35 player position exposure/risk hours $(13 \times 115$ (total games) $\times 1.33)$. The analysis was carried out using SPSS. Rates per 1000 hours were calculated taking player exposure/ risk hours into account. $\chi^{2}$ was used to assess the difference between observed and expected injuries. This figure is based on total games played - that is, game exposure time not training hours, as the ratio of training hours to games is consistent throughout the four seasons.

\section{Results}

During the study, a total of 115 games were played (table 1), and 920 injuries were recorded (232 during training and 688 during games). Per season there were $220(23.9 \%)$, $231(25.1 \%), 216(23.5 \%)$, and $253(27.5 \%)$ injuries respectively.

To evaluate differences between injuries per season, expected injury rates were calculated using player exposure/risk hours. (Reporting injuries by case number alone can often mask the true effect; however, once adjusted for exposure, true incidence figures are generated.) Both training and game injuries were examined, but exposure/risk hours were calculated by games alone because of the injury classification used in this study and because training hours were always in the same ratio to games played. Exposure/risk hours were calculated for the four seasons respectively as $605.15,622.44,397.67$, and 363.09 hours (overall 1988.35 hours). Expected injuries were then the same proportion of the total injuries as that season's exposure hours were of the total hours. Significant differences were found between the seasons at the $\mathrm{p}<0.001$ level $\left(\chi^{2}=73.22, \mathrm{df}=3\right)$, as fewer injuries were recorded than expected for the 1993/1994 and $1994 / 1995$ seasons and more injuries recorded than expected for the 1995/1996 and 1996 seasons (table 2).

When the total of 920 injuries is related to the exposure/risk hours (1988.35), it equates to 462.7 injuries per 1000 player hours. Considering game injuries alone, there were 346 injuries per 1000 player hours. Table 3 gives the rates per 1000 hours for each season. Using Spark's calculation ${ }^{6}$ of 17.33 player hours $(13 \times$ 1.33) per game, the number of injuries per

Table 3 Injury statistics per season for rates, player hours, appearances, per game and per game minutes

\begin{tabular}{|c|c|c|c|c|c|}
\hline & $1993 / 1994$ & $1994 / 1995$ & $1995 / 1996$ & 1996 & Total \\
\hline Rates per 1000 hours $(95 \% \mathrm{CI})$ & $363.6(325$ to 402$)$ & $371.1(333$ to 409$)$ & $543.2(494$ to 592$)$ & $696.8(650$ to 744$)$ & $462.7(441$ to 485$)$ \\
\hline Player hours per injury & 2.8 & 2.7 & 1.8 & 1.4 & 2.2 \\
\hline Appearances per injury & 2.1 & 2.0 & 1.4 & 1.1 & 1.6 \\
\hline No of injuries per game & 6.3 & 6.4 & 9.4 & 12.0 & 8.0 \\
\hline Minutes per injury & 12.7 & 12.5 & 8.5 & 6.7 & 10.0 \\
\hline
\end{tabular}


Table 4 Classification of injury severity using overall injuries, expressed in rates per 1000 hours (number of injury cases)

\begin{tabular}{lllrrl}
\hline Year & Transient & Minor & Moderate & \multicolumn{1}{l}{ Severe } & Total \\
\hline $1993 / 1994$ & $328.84(199)$ & $18.18(11)$ & $8.26(5)$ & $8.26(5)$ & $363.55(220)$ \\
$1994 / 1995$ & $311.68(194)$ & $32.13(20)$ & $14.46(9)$ & $12.85(8)$ & $371.12(231)$ \\
$1995 / 1996$ & $480.30(191)$ & $37.72(15)$ & $22.63(9)$ & $2.51(1)$ & $543.16(216)$ \\
1996 & $638.96(232)$ & $24.79(9)$ & $30.30(11)$ & $2.75(1)$ & $696.80(253)$ \\
\hline
\end{tabular}

Table 5 Classification of game injury severity only, expressed in rates per 1000 hours (number of injury cases)

\begin{tabular}{llllll}
\hline Year & Transient & Minor & Moderate & Severe & Total \\
\hline $1993 / 1994$ & $249.52(151)$ & $14.87(9)$ & $6.61(4)$ & $6.61(4)$ & $277.62(168)$ \\
$1994 / 1995$ & $257.05(160)$ & $22.49(14)$ & $11.25(7)$ & $9.64(6)$ & $300.43(187)$ \\
$1995 / 1996$ & $347.02(138)$ & $22.63(9)$ & $17.60(7)$ & $2.51(1)$ & $389.77(155)$ \\
1996 & $446.17(162)$ & $13.77(5)$ & $27.54(10)$ & $2.75(1)$ & $490.24(178)$ \\
\hline
\end{tabular}

hour of play across all four seasons was calculated along with the number of injuries per appearance, per game, and per game minute. Table 3 shows the results of these calculations, with a decreasing trend of player hours, player appearances, and game minutes between injuries being observed and an increasing trend of injuries per game across the seasons, especially increasing in the 1996 summer season.

Table 4 shows the increasing incidence of injury across the seasons. It highlights the increase in transient injuries and the reduction in injuries at the severe level, especially of the 1996 summer season.

To strengthen the statistics on reduced severity, surgical intervention was also observed. Of 920 cases, $19(2.1 \%)$ required surgical intervention (all related to game injuries). More injuries incurred during the two winter seasons required operation than

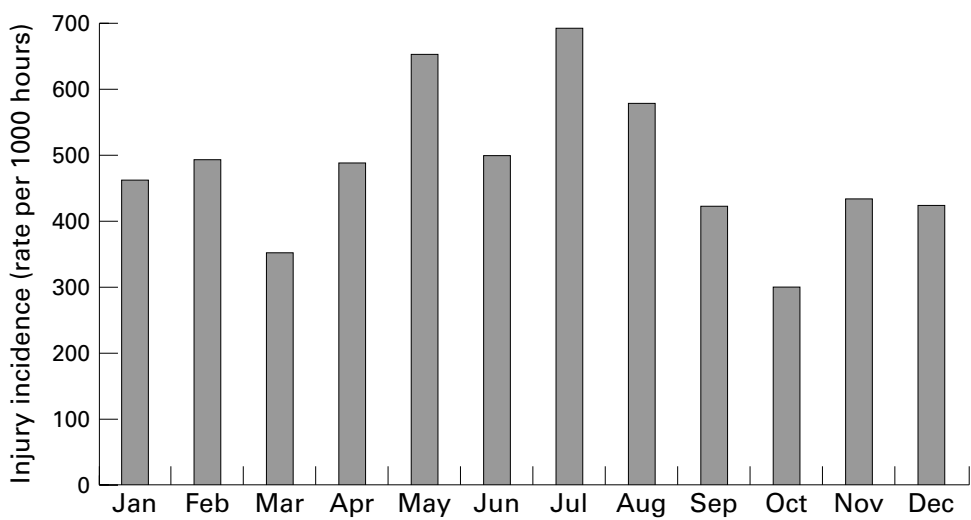

Figure 1 Overall monthly injury incidence rates.

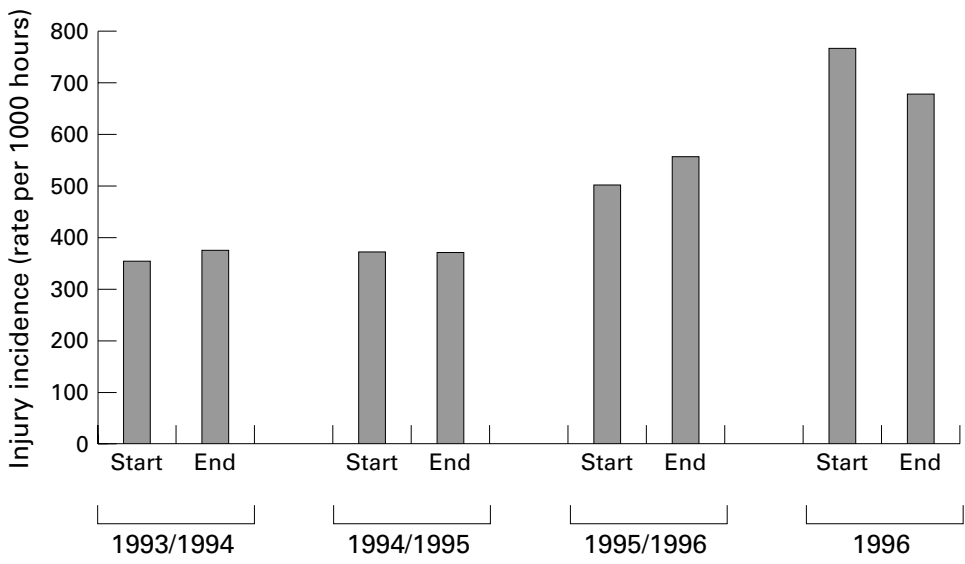

Figure 2 Incidence of injury at the start and end of each season. those incurred during the two following seasons (3.1\% (8.96 per 1000 hours) and $1.1 \%$ (4.81 per 1000 hours) respectively).

Only 104 (52.30 per 1000 player hours) injuries resulted in a game being missed. The individual season's figures are 21 (34.70 per 1000 hours), 37 (59.44 per 1000 hours), 25 (62.87 per 1000 hours), and 21 (57.84 per 1000 hours) respectively (table 4 ). Thus, even though there were fewer severe injuries (requiring five or more games to be missed), there were still a comparable number of game days lost as the result of injury in the summer 1996 season.

Considering game injuries alone, 77 (38.73 per 1000 hours) led to games being missed over the four seasons. Per season it equates to 17 (28.09 per 1000 hours), 27 (43.38 per 1000 hours), 17 (42.75 per 1000 hours), and 16 (44.07 per 1000 hours) respectively (table 5). This shows a higher incidence per 1000 hours in the 1996 summer season of game days lost despite fewer severe injuries. The different profile of injury frequencies and adjusted rates highlights the importance of considering the latter.

There was also a significant increase in training days lost in the 1996 summer season (180 per 1000 hours) in comparison with the average for the first two winter seasons and the condensed season recorded (130 and 120 per 1000 hours respectively).

As data were available on every month, injury incidence could be examined to see whether there was a pattern over the year. Unadjusted rates suggested that there were more injuries in April (135) and August (120). However, when injury cases are adjusted for number of games played, July shows the highest rate (694.04 per 1000 hours) and October the lowest (302.04 per 1000). Figure 1 suggests that the rates are highest for the "new" summer months compared with the "old" winter months.

Comparing the beginning of season with the end (by dividing each season in half), more injuries consistently occurred towards the end of the season than at the beginning. When frequencies are adjusted to rates per 1000 hours the individual seasons show no consistent pattern (fig 2).

There were significantly more game injuries than training injuries $\left(\chi^{2}=4500.7, \mathrm{df}=1\right.$, $\mathrm{p}<0.0001)$ over all four seasons, with the percentage of training injuries rising over the 
last two seasons. Even so, there were fewer training injuries than expected when training exposure hours were calculated, despite there being five times as many training sessions as games and training sessions lasting 1.5 hours and involving 22 men. Training exposure was calculated to be 18.975 exposure/risk hours (115 (total games) $\times 5$ (number training sessions per week) $\times 1.5$ (duration of each session in hours) $\times 22$ (squad of men)), which gives a training injury incidence of 12.23 per 1000 hours (232 injuries).

\section{Discussion}

The results show an increasing incidence of injury per 1000 hours over the four seasons, with the new summer league having an incidence almost twice that of the first season. This is in spite of the fact that 71 games were played in the first two seasons (exposure of 1227.59 hours) giving an incidence of 367.36 per 1000 hours and only 44 games were played in the last two seasons (exposure of 760.76 hours) giving an incidence of 619.98 per 1000 hours.

Severe injuries showed a decreasing trend from the last winter season with only one case reported in each of the last two seasons. Minor injuries also decreased in the summer season, but moderate injuries increased slightly over the seasons, with transient injuries rising in the summer season. The same applies if only game injuries are considered. This study shows that the incidence of injury increased in summer rugby but that the severity of these injuries was reduced. Of all injuries in this study, $94.8 \%$ resulted in the player taking less than one week away from training, the greatest proportion of these being from the 1996 summer season $(25.8 \%)$. However, there were more game days lost and significantly more training days lost in the 1996 summer season than the previous seasons. This shows that, even if these injuries were less severe and required less surgical intervention, they still required medical attention and led to significant time missed.

The two winter seasons were played over eight months with an average of one game per week. These seasons have similar results, with only 11 injuries more in 1994/1995 than in 1993/1994, which might be explained by the fact that one more game was played in 1994/1995. As table 3 shows, eight injuries could be expected on average per game.

The higher incidence of injury in the condensed 1995/1996 season may be explained by the short length of that season (less than 5.5 months including a three week break for the World Cup and five weeks of playing two games per week). It could be suggested that insufficient time existed between game days to allow for adequate recovery.

The summer season showed the highest injury incidence per 1000 hours ever reported in rugby league. This remains true even when all transient injuries are excluded (57.84 per 1000 hours). Other studies, in which the definition of injury was that a session/game had to be missed as a result, ${ }^{178}$ may have lost valuable data, particularly on transient/minor inju- ries. The length of the summer season was comparable with that of the condensed season (5.5 months) and actually fewer games were played, yet an injury incidence per 1000 hours that was $25 \%$ higher than that of the condensed season was observed. An explanation for this finding may be that limited time existed between the end of the condensed and the start of the summer season (nine weeks), giving insufficient time for injured players to recuperate and non-injured players to rest. This is reflected by the fact that the highest incidence of recurrent injuries was in this season (223.09 per 1000 hours). However, it could be argued that only 44 games were played in a total of 11 months, which is only eight games more and three months longer than one of the previous winter seasons. The change in time of year of the rugby league playing season introduces a warmer climate and harder unforgiving ground, which may contribute to the highly significant increase in injury incidence. The highest previously reported incidence of injury in rugby league (44.9 per 1000 hours, excluding transient injuries) ${ }^{1}$ was reported for a professional South Sydney team, who also played in warm weather on hard ground.

It has been suggested that the higher the standard of play the higher the probable incidence of injury. ${ }^{5}$ It would thus be expected that the the highest incidence of injury would be in the two winter seasons, because the team under study was then playing in the old first division with all the top clubs. However, a higher incidence of injury was observed when the team was playing in the new first division against teams of a slightly lower standard. An explanation may be that the opening statement is not justified; professional players may be at less risk of injury from tackles because of increased expertise. Another explanation could lie in the style, time, and environment of the last two seasons, condensed and summer, which led to the increased incidence of injury along with the decreased skill of the new division teams. However, six teams were "relegated" to allow the formation of the Super League and some players stayed with each of the "relegated teams", and therefore standards of play were not significantly reduced. Comparing these data with a previous study, ${ }^{5}$ which looked at an "old" second division side and found a lower incidence rate which included game and training injuries, would suggest that it is not relegation that led to the increased incidence found in this study.

This study agrees with others ${ }^{9}{ }^{10}$ that showed peak injury frequency towards the end of the season; however, when adjusted for rates, no consistent pattern was found. In each season, more games were played in the later months. Considering monthly incidence rates, July showed the highest individual monthly rate recorded and only appeared once in the "new" summer season. August had the third highest rate and appeared in every season. The "new" summer months showed a high rate of incidence in comparison with previous autumn 
and winter months (fig 1). The harder ground and warmer weather may explain these findings.

The incidence rate found in this study of 462.7 per 1000 player hours is considerably higher than previously reported using both training and game injuries. ${ }^{78}$ This study included all injuries even if they did not lead to game/training days being missed, which may account for the higher incidence, as, in all four years, transient injuries made up $82-92 \%$ of the total injuries per season. The above mentioned studies ${ }^{78}$ only included transient injuries that led to game/training being missed. Taking game injuries alone, this study still has a higher incidence (346.02 per 1000 hours) than a study using similar methodology (277.78 per 1000 hours). ${ }^{4}$

When considering injuries resulting in games being missed, this study has a high incidence (52.3 per 1000 hours) compared with other reports (44.9 per 1000 hours $^{1}$ and 34 per 1000 hours $\left.{ }^{8}\right)$. The higher incidence than the former ${ }^{1}$ may be explained by the fact that no training or transient injuries were included, which, if extrapolated to this study, gives an incidence of 38.73 per 1000 hours. This is lower than the former but higher than the latter calculation based on the same criteria. Looking at the 1996 summer season excluding training and transient injuries, it equates to 44.07 per 1000 hours, which is very comparable with the former Australian study. ${ }^{1}$

Throughout all four seasons, significantly more injuries occurred during matches, but in the last two seasons the percentage of training injuries was rising. This may reflect that one of the seasons was condensed and ran almost immediately into the next, thus increasing the risk of a training injury. These findings are consistent with other studies, but the training injuries recorded in each season are higher than previously reported. An explanation could be that, for this study, the physiotherapist was present at every training session and was thus able to document every injury incident.

In conclusion, an increased incidence of injury was observed in summer rugby, but most of these injuries were below the severe level and fewer required surgery. Significantly more cases of injury led to training days being lost. These findings may have been confounded by the short break between the condensed and summer seasons. The effect of this increased incidence and the training time lost on a player and his career is yet to be determined. Further injury data should be collected to determine the true effect of summer rugby.

We would like to thank everyone who helped with this study. We are grateful to $\mathrm{Mr} \mathrm{C}$ Gissane and the Centre for Sports
Medicine, Nottingham for their support. Special thanks go to Featherstone Rovers RLFC, especially the players, for support over the last four years and for providing the opportunity to collect the data.

1 Gibbs N. Injuries in professional rugby league. A 3 year prospective study. Am $\mathcal{F}$ Sports Med 1993;21:696-700.

2 Meir R, Arthur D, Forrest M. Time and motion analysis of professional rugby league: a case study. Strength $\mathbb{E}$ Conditioning Coach 1993;3:24-9.

3 Larder P. The rugby league coaching manual. New ed. London: Kingswood Press, 1992.

4 Alexander D, Kennedy M, Kennedy J. Injuries in rugby league football. Med $\mathcal{F}$ Aust 1979;2:341-2.

5 Stephenson S, Gissane C, Jennings D. Injury in rugby league: a four year prospective study. Brf Sports Med 1996; 30:331-4

6 Sparks JP. Half a million hours of rugby football. Br 7 Sports Med 1981;15:30-2.

7 Seward H, Orchard J, Hazard H, Collinson D. Football injuries in Australia at the elite level. Med f Aust 1993;159: 298-301.

8 Gissane C, Jennings D, Standing P. Incidence of injury in rugby league football. Physiotherapy 1993;79:1-6.

9 Alexander D, Kennedy M, Kennedy J. Rugby league Alexander D, Kennedy M, Kennedy J. Rugby league
football injuries over 2 competitive seasons. Med $\mathcal{f}$ Aust football injuries

10 Taylor TKF, Coolican MRJ. Spinal cord injuries in Australian footballers 1960-1985. Med F Aust 1987;147:112-18.

11 Garraway M, Macleod D. Epidemiology of rugby football injuries. Lancet 1995;345:1485-7. 